\title{
Multi-parameter second-order impulsive indefinite boundary value problems
}

\author{
Lishuai Jiao ${ }^{1}$ and Xuemei Zhang ${ }^{1 *}$
}

\footnotetext{
"Correspondence: zxm74@sina.com 'Department of Mathematics and Physics, North China Electric Power University, Beijing, People's Republic of China
}

\begin{abstract}
We consider the solvable intervals of three positive parameters $\lambda_{i}(i=1,2,3)$ in which the second-order impulsive boundary value problem

$$
\begin{cases}-x^{\prime \prime}=a(t) x y+\lambda_{1} g(t) f(x), & 0<t<1, t \neq t_{k}, \\ -y^{\prime \prime}=\lambda_{2} b(t) x, & 0<t<1, \\ \left.\Delta x\right|_{t=t_{k}}=\lambda_{3} l_{k}\left(x\left(t_{k}\right)\right), & k=1,2, \ldots, n, \\ x(0)=0, \quad x^{\prime}(1)=0, & \\ y(0)=y(1)=0 & \end{cases}
$$

admits at least two positive solutions. The main interest is that the weight functions $a(t), b(t)$, and $g(t)$ change sign on $[0,1], \lambda_{i}(i=1,2,3) \not \equiv 1$, and $I_{k} \neq 0(k=1,2, \ldots, n)$. We will obtain several interesting results: there exist positive constants $\lambda^{*}, \lambda_{*}, \lambda_{i}^{*}(i=1,3)$, $\lambda_{i}^{* *}(i=1,2,3)$ and $\alpha$ with $\alpha \neq 1$ such that: (i) if $\alpha>1$, then for $\lambda_{i} \in\left[\lambda_{i}^{*},+\infty\right)(i=1,3)$ and $\lambda_{2} \in\left[\lambda_{*}, \lambda^{*}\right]$, the above boundary value problem admits at least two positive solutions; (ii) if $0<\alpha<1$, then for $\lambda_{i} \in\left(0, \lambda_{i}^{* *}\right](i=1,2,3)$, the above boundary value problem admits at least two positive solutions.
\end{abstract}

Keywords: Solvable intervals of three parameters; Positive solutions; Indefinite impulsive boundary value problem; Fixed point technique

\section{Introduction}

It is well established that impulsive differential equations can provide a natural description of observed evolution processes. Therefore it is regarded as an important mathematical tool to better understand many real world problems in applied sciences. On details and explanations, we refer the readers to the texts [1-4]. Specially, Nieto and O'Regan [5] pointed out that in a second order differential equation $u^{\prime \prime}=f\left(t, u, u^{\prime}\right)$, one usually considers impulses in the position $u$ and the velocity $u^{\prime}$. However, in the motion of spacecraft one has to consider instantaneous impulses depending on the position that result in jump discontinuities in velocity, but with no change in position [6]. The impulses only on velocity occur also in impulsive mechanics [7]. Recently, a great interest has been shown by many authors in the subject of impulsive boundary value problems (IBVPs) by applying different approaches, for example, see [8-28] and the references cited therein.

At the same time, we notice that a class of indefinite weight problems have attracted the attention of Ma and Han [29], López-Gómez and Tellini [30], Boscaggin and Zanolin [31,

(c) The Author(s) 2018. This article is distributed under the terms of the Creative Commons Attribution 4.0 International License (http://creativecommons.org/licenses/by/4.0/), which permits unrestricted use, distribution, and reproduction in any medium, provided you give appropriate credit to the original author(s) and the source, provide a link to the Creative Commons license, and indicate if changes were made. 
32], Sovrano and Zanolin [33], Bravo and Torres [34], Wang and An [35], and Yao [36]. In [29], Ma and Han considered the following boundary value problem:

$$
\left\{\begin{array}{l}
u^{\prime \prime}+\lambda a(t) f(u)=0, \quad 0<t<1, \\
u(0)=u(1)=0
\end{array}\right.
$$

where $a \in C[0,1]$ may change sign, $\lambda$ is a parameter. They proved the existence, multiplicity, and stability of positive solutions for the above problem by applying bifurcation techniques.

In [33], applying the shooting method, Sovrano and Zanolin presented a multiplicity result of positive solutions for the Neumann problem

$$
\begin{cases}u^{\prime \prime}+a(t) f(u)=0, & 0<t<1, \\ u(t)>0, & t \in[0, T], \\ u^{\prime}(0)=u^{\prime}(T)=0, & \end{cases}
$$

where the weight function $a \in C[0,1]$ has indefinite sign.

Recently, Wang and An in [35] dealt with the existence and multiplicity of positive solutions for the second-order differential system

$$
\begin{cases}-u^{\prime \prime}=a(t) \varphi u+h(t) f(u), & 0<t<1, \\ -\varphi^{\prime \prime}=b(t) u, & 0<t<1, \\ u(0)=u(1)=0, & \\ \varphi(0)=\varphi(1)=0, & \end{cases}
$$

where $a(t), b(t), g(t)$ are allowed to change sign on $[0,1]$.

Very recently, López-Gómez, Omari, and Rivetti $[37,38]$ studied a class of quasilinear indefinite problems

$$
\left\{\begin{array}{l}
-\left(\frac{u^{\prime}}{\sqrt{1+u^{\prime 2}}}\right)^{\prime}=\lambda a(x) f(u), \\
u^{\prime}(0)=u^{\prime}(1)=0,
\end{array}\right.
$$

where $\lambda \in \mathcal{R}$ is a parameter, $a \in L^{1}[0,1]$ changes sign. They derived many results on the existence and the multiplicity of positive (regular) solutions by applying topological degree and variational approach, respectively.

For all we know, in literature there are no articles on multiple positive solutions for the analogous of indefinite impulsive problems with multiple parameters. More precisely, the study of $\lambda_{i}(i=1,2,3) \not \equiv 1$, and $a(t), b(t)$, and $g(t)$ changing sign on $[0,1]$ is still open for the second order impulsive boundary value problem

$$
\begin{cases}-x^{\prime \prime}=a(t) x y+\lambda_{1} g(t) f(x), & 0<t<1, t \neq t_{k}, \\ -y^{\prime \prime}=\lambda_{2} b(t) x, & 0<t<1, \\ \left.\Delta x\right|_{t=t_{k}}=\lambda_{3} I_{k}\left(x\left(t_{k}\right)\right), & k=1,2, \ldots, n, \\ x(0)=0, \quad x^{\prime}(1)=0, & \\ y(0)=y(1)=0, & \end{cases}
$$


where $\lambda_{1}>0, \lambda_{2}>0$, and $\lambda_{3}>0$ are three parameters, $a(t), b(t), g(t)$ change sign on $[0,1]$, $t_{k}(k=1,2, \ldots, n)$ (where $n$ is a fixed positive integer) are fixed points with $0=t_{0}<t_{1}<$ $t_{2}<\cdots<t_{k}<\cdots<t_{n}<t_{n+1}=1,\left.\Delta x\right|_{t=t_{k}}$ denotes the jump of $x(t)$ at $t=t_{k}$, i.e., $\left.\Delta x\right|_{t=t_{k}}=$ $x\left(t_{k}^{+}\right)-x\left(t_{k}^{-}\right)$, where $x\left(t_{k}^{+}\right)$and $x\left(t_{k}^{-}\right)$represent the right-hand limit and left-hand limits of $x(t)$ at $t=t_{k}$, respectively. Moreover, throughout this paper, we assume that $a, g, b, f$ and $I_{k}(k=1,2, \ldots, n)$ satisfy

$\left(H_{1}\right) a, g, b:[0,1] \rightarrow(-\infty,+\infty)$ and continuous, and there exists a constant $\xi \in(0,1)$ such that

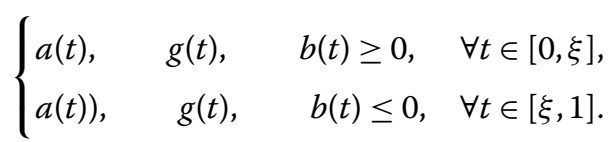

Moreover, $a(t), g(t), b(t)$ do not vanish identically on any subinterval of $[0,1]$.

$\left(H_{2}\right) f \in C\left(\mathcal{R}^{+}, \mathcal{R}^{+}\right)$, and $f(s)>0$ for $s>0$, where $\mathcal{R}^{+}=[0,+\infty)$.

$\left(H_{3}\right) I_{k} \in C\left(\mathcal{R}^{+}, \mathcal{R}^{+}\right)$, and $I_{k}(s)>0$ for $s>0, k=1,2, \ldots, n$.

We denote

$a^{+}(t)=\max \{a(t), 0\}, a^{-}(t)=-\min \{a(t), 0\}$ such that $a(t)=a^{+}(t)-a^{-}(t), \forall t \in[0,1]$,

$g^{+}(t)=\max \{g(t), 0\}, g^{-}(t)=-\min \{g(t), 0\}$ such that $g(t)=g^{+}(t)-g^{-}(t), \forall t \in[0,1]$,

$b^{+}(t)=\max \{b(t), 0\}, b^{-}(t)=-\min \{b(t), 0\}$ such that $b(t)=b^{+}(t)-b^{-}(t), \forall t \in[0,1]$.

Some special cases of problem (1.1) have been investigated. For example, Sovrano and Zanolin [33] considered problem (1.1) when $\lambda_{1} \equiv 1, \lambda_{2}=0$, and $I_{k}=0(k=1,2, \ldots, n)$. By applying the shooting method, they presented a multiplicity result of positive solutions for problem (1.1). In [35], Wang and An studied problem (1.1) with $\lambda_{1} \equiv 1, \lambda_{2} \equiv 1$, and $I_{k}=0(k=1,2, \ldots, n)$. Using a fixed point technique, they showed the existence of positive solutions for problem (1.1).

Remark 1.1 Comparing with other related indefinite problems [29-32], the main features of this paper are as follows.

(i) $I_{k} \neq 0(k=1,2, \ldots, n)$ is considered.

(ii) $\lambda_{i}>0$ is available, not only $\lambda_{i} \equiv 1, i=1,2,3$.

(iii) The method used in the present paper is completely different from those in [29-32].

Remark 1.2 It is very difficult to obtain the solvable intervals of three positive parameters $\lambda_{i}(i=1,2,3)$, especially for parameter $\lambda_{2}$. For details, see the proof of part I of Theorem 3.1.

It is well accepted that fixed point theorems in cones have been instrumental in showing the existence, multiplicity of positive solutions of various boundary value problems for differential equations. See, for instance, [39-46] and the references therein. In this paper, we will use Krasnoselskii's fixed point theorem in a cone to investigate the existence and multiplicity of positive solutions of problem (1.1). We remark that this is probably the first time that multiple positive solutions of impulsive differential system with indefinite weight and multiple parameters have been studied.

The rest of the present article is organized as follows. In Sect. 2 we give some preliminary results to be used in the subsequent sections. Section 3 is devoted to stating and proving the main results. Several related remarks are given in Sect. 4 , and we give an example to illustrate the main results in the final section. 


\section{Preliminaries}

In this section, we give some preliminary results for the convenience of later use and reference.

$$
\begin{aligned}
& \text { Let } J=[0,1], J^{\prime}=J \backslash\left\{t_{1}, t_{2}, \ldots, t_{n}\right\}, J_{0}=\left[t_{0}, t_{1}\right], J_{k}=\left(t_{k}, t_{k+1}\right], k=1,2, \ldots, n \text {, and } \\
& \qquad P C[0,1]=\left\{x: x \text { is continuous at } t \neq t_{k}, x\left(t_{k}^{-}\right)=x\left(t_{k}\right) \text { and } x\left(t_{k}^{+}\right) \text {exists, } k=1,2, \ldots, n\right\} .
\end{aligned}
$$

Then $P C[0,1]$ is a real Banach space with the norm

$$
\|x\|_{P C}=\max _{t \in J}|x(t)|
$$

By a positive solution of problem (1.1), we understand a pair of functions $(x, y)$ with $x \in C^{2}\left(J^{\prime}\right) \cap P C[0,1], y \in C^{2}(J) \cap C[0,1]$, if $(x, y)$ satisfies problem (1.1), and $x \geq 0, t \in J^{\prime}$, $y \geq 0, t \in J, x, y \neq 0$.

It is clear that problem (1.1) is equivalent to the following two boundary value problems:

$$
\begin{cases}-x^{\prime \prime}=a(t) x y+\lambda_{1} g(t) f(x), & 0<t<1, t \neq t_{k}, \\ \left.\Delta x\right|_{t=t_{k}}=\lambda_{3} I_{k}\left(x\left(t_{k}\right)\right), & k=1,2, \ldots, n, \\ x(0)=0, \quad x^{\prime}(1)=0, & \end{cases}
$$

and

$$
\left\{\begin{array}{l}
-y^{\prime \prime}=\lambda_{2} b(t) x, \quad 0<t<1, \\
y(0)=y(1)=0 .
\end{array}\right.
$$

Lemma 2.1 Assume that $\left(H_{1}\right)-\left(H_{3}\right)$ hold. Then problem (2.1) has a solution $x$, and $x$ can be expressed in the form:

$$
\begin{aligned}
x(t)= & \int_{0}^{1} G(t, s) a(s) x(s) y(s) d s+\lambda_{1} \int_{0}^{1} G(t, s) g(s) f(x(s)) d s \\
& +\lambda_{3} \sum_{k=1}^{n} G_{s}^{\prime}\left(t, t_{k}\right) I_{k}\left(x\left(t_{k}\right)\right),
\end{aligned}
$$

where

$$
G(t, s)= \begin{cases}t, & 0 \leq t \leq s \leq 1 \\ s, & 0 \leq s \leq t \leq 1\end{cases}
$$

It is obvious that

$$
G_{s}^{\prime}(t, s)= \begin{cases}0, & 0 \leq t \leq s \leq 1 \\ 1, & 0 \leq s \leq t \leq 1\end{cases}
$$

and

$$
\max _{t, s \in J, t \neq s}\left|G_{s}^{\prime}(t, s)\right|=1
$$


Proof The proof of Lemma 2.1 is similar to that of Lemma 2.6 in [47].

It follows from the definition of $G(t, s)$ that Proposition 2.1 holds.

Proposition 2.1 Let $G(t, s)$ be given as in (2.4). Then we have the following results:

$$
\begin{aligned}
& G(t, s)>0, \quad \forall t, s \in(0,1), \\
& t G(s, s) \leq G(t, s) \leq G(s, s)=s \leq 1, \quad \forall t, s \in J, \\
& G(t, s) \geq \xi G(s, s), \quad \forall t \in[\xi, 1], s \in J .
\end{aligned}
$$

Proof By the definition of $G(t, s)$, it is not difficult to see that $G(t, s)>0, \forall t, s \in(0,1)$.

Next, turning to (2.6), if $t \leq s$, then $G(t, s)=t \leq s$; if $t \geq s$, then $G(t, s)=s$. So we get that $G(t, s) \leq s=G(s, s), \forall t, s \in J$.

On the other hand, noticing that $t, s \in J$, if $t \leq s$, then $G(t, s)=t \geq t$; if $t \geq s$, then $G(t, s)=$ $s \geq s t$. Therefore, we obtain that $G(t, s) \geq t s=t G(s, s), \forall t, s \in J$. This gives the proof of (2.6).

If $t \in[\xi, 1]$, it is easy to see that by (2.6), $G(t, s) \geq \xi G(s, s), \forall s \in J$.

Remark 2.1 Noticing that $t_{k} \in(0,1)$, we get $G\left(t_{k}, t_{k}\right)>0$.

Lemma 2.2 Assume that $\left(H_{1}\right)-\left(H_{3}\right)$ hold. Then problem (2.2) has a solution y given by

$$
y(t)=\lambda_{2} \int_{0}^{1} G_{1}(t, s) b(s) x(s) d s
$$

where

$$
G_{1}(t, s)= \begin{cases}t(1-s), & 0 \leq t \leq s \leq 1 \\ s(1-t), & 0 \leq s \leq t \leq 1\end{cases}
$$

Proof The proof of Lemma 2.2 is similar to that of Lemma 2.1 in [48].

Similar to the proof of Proposition 2.1, we can get Proposition 2.2.

Proposition 2.2 Let $G_{1}(t, s)$ be given as in (2.8). Then we have the following results:

$$
\begin{aligned}
& G_{1}(t, s)>0, \quad \forall t, s \in(0,1), \\
& G_{1}(t, t) G_{1}(s, s) \leq G_{1}(t, s) \leq G_{1}(s, s)=s(1-s) \leq \frac{1}{4}, \quad \forall t, s \in J, \\
& G_{1}(t, s) \geq \xi^{2} G_{1}(s, s), \quad \forall t \in[\xi, 1-\xi], s \in J .
\end{aligned}
$$

Remark 2.2 Letting $(x, y)$ be a solution of problem (1.1), from Lemma 2.1 and Lemma 2.2, we have

$$
\begin{aligned}
x(t)= & \lambda_{2} \int_{0}^{1} \int_{0}^{1} G(t, s) G_{1}(s, \tau) a(s) b(\tau) x(s) x(\tau) d \tau d s \\
& +\lambda_{1} \int_{0}^{1} G(t, s) g(s) f(x(s)) d s+\lambda_{3} \sum_{k=1}^{n} G_{s}^{\prime}\left(t, t_{k}\right) I_{k}\left(x\left(t_{k}\right)\right),
\end{aligned}
$$


and $y$ is defined in (2.7).

To obtain the existence of positive solution of problem (1.1), we make the following hypotheses:

$\left(H_{4}\right)$ There exists $0<\sigma_{1}<\xi$ such that

$$
\sigma_{1} \int_{\sigma_{1}}^{\xi} G_{1}(t, s) b^{+}(s) d s \geq \xi \int_{\xi}^{1} G_{1}(t, s) b^{-}(s) d s
$$

$\left(H_{5}\right)$ There exists $0<\sigma_{2}<\xi$ such that

$$
\sigma_{2} \int_{\sigma_{2}}^{\xi} G(t, s) G_{1}(s, s) a^{+}(s) d s \geq \xi \int_{\xi}^{1} G(t, s) a^{-}(s) d s
$$

$\left(H_{6}\right)$ There exists $0<\mu \leq 1$ such that

$$
f(\omega) \geq \mu \varphi(\omega), \quad \omega \in[0,+\infty)
$$

where $\varphi(\omega)=\max \{f(\rho): 0 \leq \rho \leq \omega\}$;

$\left(H_{7}\right)$ There exist $0<\alpha<+\infty$ with $\alpha \neq 1$ and $k_{1}, k_{2}, l_{1}, l_{2}>0$ such that

$$
k_{1} x^{\alpha} \leq f(x) \leq k_{2} x^{\alpha}, \quad l_{1} x^{\alpha} \leq I_{k}(x) \leq l_{2} x^{\alpha}, \quad x \in[0,+\infty)
$$

$\left(H_{8}\right)$ There exists $0<\sigma_{3}<\xi$ satisfying $\frac{\sigma_{3}}{2}<t_{1}<\sigma_{3}$ such that

$$
\sigma_{3}^{\alpha} \mu^{2} k_{1} \int_{\sigma_{3}}^{\xi} G(t, s) g^{+}(s) d s \geq k_{2} \xi^{\alpha} \int_{\xi}^{1} G(t, s) g^{-}(s) d s .
$$

We denote

$$
\begin{aligned}
& C_{0}^{+}[0,1]=\left\{x \in P C[0,1]: \min _{t \in J} x(t) \geq 0, x(0)=0, x^{\prime}(1)=0\right\}, \\
& K=\left\{x \in C_{0}^{+}[0,1]: x \text { is concave on }[0, \xi], \text { and convex on }[\xi, 1]\right\} .
\end{aligned}
$$

If $x \in K$, it is not difficult to see that $\|x\|_{P C}=\max _{0 \leq t \leq \xi} x(t)$. Also, for a positive number $r$, we define $\Omega_{r}$ by $\Omega_{r}=\left\{x \in K:\|x\|_{P C}<r\right\}$, and then we get $\partial \Omega_{r}=\left\{x \in K:\|x\|_{P C}=r\right\}$.

Define $T: K \rightarrow P C[0,1]$ by

$$
\begin{aligned}
(T x)(t)= & \lambda_{2} \int_{0}^{1} \int_{0}^{1} G(t, s) G_{1}(s, \tau) a(s) b(\tau) x(s) x(\tau) d \tau d s \\
& +\lambda_{1} \int_{0}^{1} G(t, s) g(s) f(x(s)) d s+\lambda_{3} \sum_{k=1}^{n} G_{s}^{\prime}\left(t, t_{k}\right) I_{k}\left(x\left(t_{k}\right)\right) .
\end{aligned}
$$

Remark 2.3 It follows from Lemmas 2.1-2.2 and Remark 2.2 that $(x, y)$ is a solution of problem (1.1) if and only if $x$ is a fixed point of operator $T$ and $y$ satisfies (2.7).

Lemma 2.3 Assume that $\left(H_{1}\right)-\left(H_{8}\right)$ hold. Then $T(K) \subset K$ and $T: K \rightarrow K$ is completely continuous. 
Proof Define a function $e:[0,1] \rightarrow[0,1]$ as follows:

$$
\begin{aligned}
& \text { if } x(1)=0, \text { then } e(t)=\min \left\{\frac{t}{\xi}, \frac{1-t}{1-\xi}\right\}, \quad \forall t \in J ; \\
& \text { if } x(1)>0 \text {, then } e(t)=\min \left\{\frac{t}{\xi}, 1\right\}, \quad \forall t \in J .
\end{aligned}
$$

So, we get $\min _{\sigma \leq t \leq \xi} e(t)=\frac{\sigma}{\xi}, \max _{\xi \leq t \leq 1} e(t)=1$.

Letting $x \in K$, then $x$ is concave on $[0, \xi]$ and convex on $[\xi, 1]$. Noticing that $x(0)=0$, $x^{\prime}(1)=0$, we get $x(t) \geq x(\xi) e(t), t \in[0, \xi], x(t) \leq x(\xi) e(t), t \in[\xi, 1]$.

Firstly, for any $x \in K$, we show that

$$
\int_{0}^{1} G_{1}(t, s) b(s) x(s) d s \geq \int_{0}^{\sigma_{1}} G_{1}(t, s) b^{+}(s) x(s) d s, \quad t \in J .
$$

In fact, for $x \in K$, noticing $\left(H_{4}\right)$, we get

$$
\begin{aligned}
\int_{0}^{1} & G_{1}(t, s) b(s) x(s) d s-\int_{0}^{\sigma_{1}} G_{1}(t, s) b^{+}(s) x(s) d s \\
& =\int_{\sigma_{1}}^{\xi} G_{1}(t, s) b^{+}(s) x(s) d s-\int_{\xi}^{1} G_{1}(t, s) b^{-}(s) x(s) d s \\
& \geq \int_{\sigma_{1}}^{\xi} G_{1}(t, s) b^{+}(s) e(s) x(\xi) d s-\int_{\xi}^{1} G_{1}(t, s) b^{-}(s) e(s) x(\xi) d s \\
& \geq x(\xi)\left[\min _{s \in\left[\sigma_{1}, \xi\right]} e(s) \int_{\sigma_{1}}^{\xi} G_{1}(t, s) b^{+}(s) d s-\max _{s \in[\xi, 1]} e(s) \int_{\xi}^{1} G_{1}(t, s) b^{-}(s) d s\right] \\
& =x(\xi)\left[\frac{\sigma_{1}}{\xi} \int_{\sigma_{1}}^{\xi} G_{1}(t, s) b^{+}(s) d s-\int_{\xi}^{1} G_{1}(t, s) b^{-}(s) d s\right] \\
& \geq 0,
\end{aligned}
$$

which shows that (2.12) holds.

Next, for any $x \in K$, we prove

$$
\begin{aligned}
& \int_{0}^{1} \int_{0}^{1} G(t, s) G_{1}(s, \tau) a(s) b(\tau) x(s) x(\tau) d \tau d s \\
& \quad \geq \int_{0}^{\sigma_{2}} \int_{0}^{1} G(t, s) G_{1}(s, \tau) a^{+}(s) b(\tau) x(s) x(\tau) d \tau d s, \quad t \in J .
\end{aligned}
$$

Noticing $\int_{0}^{1} G_{1}(t, s) b(s) x(s) d s \geq 0, \forall t \in J,(2.9)$ and $\left(H_{5}\right)$, we obtain

$$
\begin{aligned}
\int_{0}^{1} & \int_{0}^{1} G(t, s) G_{1}(s, \tau) a(s) b(\tau) x(s) x(\tau) d \tau d s \\
& -\int_{0}^{\sigma_{2}} \int_{0}^{1} G(t, s) G_{1}(s, \tau) a^{+}(s) b(\tau) x(s) x(\tau) d \tau d s \\
& =\int_{\sigma_{2}}^{\xi} \int_{0}^{1} G(t, s) G_{1}(s, \tau) a^{+}(s) b(\tau) x(s) x(\tau) d \tau d s
\end{aligned}
$$




$$
\begin{aligned}
& -\int_{\xi}^{1} \int_{0}^{1} G(t, s) G_{1}(s, \tau) a^{-}(s) b(\tau) x(s) x(\tau) d \tau d s \\
\geq & \int_{\sigma_{2}}^{\xi} G(t, s) a^{+}(s) e(s) x(\xi) \int_{0}^{1} G_{1}(s, \tau) b(\tau) x(\tau) d \tau d s \\
& -\int_{\xi}^{1} G(t, s) a^{-}(s) e(s) x(\xi) \int_{0}^{1} G_{1}(s, \tau) b(\tau) x(\tau) d \tau d s \\
\geq & \int_{\sigma_{2}}^{\xi} G(t, s) a^{+}(s) \min _{s \in\left[\sigma_{2}, \xi\right]} e(s) x(\xi) \int_{0}^{1} G_{1}(s, \tau) b(\tau) x(\tau) d \tau d s \\
& -\int_{\xi}^{1} G(t, s) a^{-}(s) \max _{s \in[\xi, 1]} e(s) x(\xi) \int_{0}^{1} G_{1}(s, \tau) b(\tau) x(\tau) d \tau d s \\
\geq & x(\xi) \frac{\sigma_{2}}{\xi} \int_{\sigma_{2}}^{\xi} G(t, s) a^{+}(s) \int_{0}^{1} G_{1}(s, \tau) b(\tau) x(\tau) d \tau d s \\
& -x(\xi) \int_{\xi}^{1} G(t, s) a^{-}(s) \int_{0}^{1} G_{1}(s, \tau) b(\tau) x(\tau) d \tau d s \\
\geq & x(\xi) \frac{\sigma_{2}}{\xi} \int_{\sigma_{2}}^{\xi} G(t, s) a^{+}(s) \int_{0}^{1} G_{1}(s, s) G_{1}(\tau, \tau) b(\tau) x(\tau) d \tau d s \\
& -x(\xi) \int_{\xi}^{1} G(t, s) a^{-}(s) \int_{0}^{1} G_{1}(\tau, \tau) b(\tau) x(\tau) d \tau d s \\
= & x(\xi) \int_{0}^{1} G_{1}(\tau, \tau) b(\tau) x(\tau) d \tau\left[\frac{\sigma_{2}}{\xi} \int_{\sigma_{2}}^{\xi} G(t, s) G_{1}(s, s) a^{+}(s) d s-\int_{\xi}^{1} G(t, s) a^{-}(s) d s\right],
\end{aligned}
$$

which proves that (2.13) holds.

Thirdly, for any $x \in K$, we prove

$$
\int_{0}^{1} G(t, s) g(s) f(x(s)) d s \geq \int_{0}^{\sigma_{3}} G(t, s) g^{+}(s) f(x(s)) d s, \quad t \in J .
$$

In fact, for any $t \in J$ and $x \in K$, noticing (2.6), it is obvious that by $\left(H_{6}\right)-\left(H_{8}\right)$

$$
\begin{aligned}
& \int_{0}^{1} G(t, s) g(s) f(x(s)) d s-\int_{0}^{\sigma_{3}} G(t, s) g^{+}(s) f(x(s)) d s \\
& \quad=\int_{\sigma_{3}}^{\xi} G(t, s) g^{+}(s) f(x(s)) d s-\int_{\xi}^{1} G(t, s) g^{-}(s) f(x(s)) d s \\
& \quad \geq \mu \int_{\sigma_{3}}^{\xi} G(t, s) g^{+}(s) \varphi(u(s)) d s-\int_{\xi}^{1} G(t, s) g^{-}(s) \varphi(u(s)) d s \\
& \quad \geq \mu \int_{\sigma_{3}}^{\xi} G(t, s) g^{+}(s) \varphi(e(s) u(\xi)) d s-\int_{\xi}^{1} G(t, s) g^{-}(s) \varphi(e(s) u(\xi)) d s \\
& \geq \mu \int_{\sigma_{3}}^{\xi} G(t, s) g^{+}(s) f(e(s) u(\xi)) d s-\frac{1}{\mu} \int_{\xi}^{1} G(t, s) g^{-}(s) f(e(s) u(\xi)) d s \\
& \quad \geq \mu \int_{\sigma_{3}}^{\xi} G(t, s) g^{+}(s) k_{1} e^{\alpha}(s) u^{\alpha}(\xi) d s-\frac{1}{\mu} \int_{\xi}^{1} G(t, s) g^{-}(s) k_{2} e^{\alpha}(s) u^{\alpha}(\xi) d s \\
& \geq \mu\left[\min _{s \in\left[\sigma_{3}, \xi\right]} e(s)\right]^{\alpha} \int_{\sigma_{3}}^{\xi} G(t, s) g^{+}(s) k_{1} u^{\alpha}(\xi) d s
\end{aligned}
$$




$$
\begin{aligned}
& -\frac{1}{\mu}\left[\min _{s \in[\xi, 1]} e(s)\right]^{\alpha} \int_{\xi}^{1} G(t, s) g^{-}(s) k_{2} u^{\alpha}(\xi) d s \\
\geq & u^{\alpha}(\xi)\left[\mu k_{1} \frac{\sigma_{3}^{\alpha}}{\xi^{\alpha}} \int_{\sigma_{3}}^{\xi} G(t, s) g^{+}(s) d s\right. \\
& \left.-\frac{1}{\mu} k_{2} \int_{\xi}^{1} G(t, s) g^{-}(s) d s\right] \\
\geq & 0 .
\end{aligned}
$$

This proves that (2.14) holds. Thus, for $x \in K$, we get

$$
\begin{aligned}
(T x)(t)= & \lambda_{2} \int_{0}^{1} \int_{0}^{1} G(t, s) G_{1}(s, \tau) a(s) b(\tau) x(s) x(\tau) d \tau d s \\
& +\lambda_{1} \int_{0}^{1} G(t, s) g(s) f(x(s)) d s+\lambda_{3} \sum_{k=1}^{n} G_{s}^{\prime}\left(t, t_{k}\right) I_{k}\left(x\left(t_{k}\right)\right) \\
\geq & \lambda_{2} \int_{0}^{\sigma_{2}} G(t, s) a^{+}(s) x(s) \int_{0}^{1} G_{1}(s, \tau) b(\tau) x(\tau) d \tau d s \\
& +\lambda_{1} \int_{0}^{\sigma_{3}} G(t, s) g^{+}(s) f(x(s)) d s+\lambda_{3} \sum_{k=1}^{n} G_{s}^{\prime}\left(t, t_{k}\right) I_{k}\left(x\left(t_{k}\right)\right) \\
\geq & 0 .
\end{aligned}
$$

Moreover, by direct calculation, we obtain

$$
\begin{aligned}
& (T x)(0)=0, \quad(T x)^{\prime}(1)=0 \\
& (T x)^{\prime \prime}(t)=-\lambda_{2} a^{+}(t) x(t) \int_{0}^{1} G_{1}(t, s) b(s) x(s) d s-\lambda_{1} g^{+}(t) f(x) \leq 0, \quad t \in[0, \xi], \\
& (T x)^{\prime \prime}(t)=\lambda_{2} a^{-}(t) x(t) \int_{0}^{1} G_{1}(t, s) b(s) x(s) d s+\lambda_{1} g^{-}(t) f(x) \geq 0, \quad t \in[\xi, 1]
\end{aligned}
$$

which shows that $T x$ is concave on $[0, \xi]$ and convex on $[\xi, 1]$. It follows that $T: K \rightarrow K$.

Finally, by standard methods and Arzelà-Ascoli theorem, one can prove $T$ is completely continuous. So it is omitted.

Remark 2.4 The idea of the proof of Lemma 2.3 comes from Theorem 3.1 of [36].

Lemma 2.4 (Theorem 2.3.4 of [49], Fixed point theorem of cone expansion and compression of norm type) Let $\Omega_{1}$ and $\Omega_{2}$ be two bounded open sets in a real Banach space E such that $0 \in \Omega_{1}$ and $\bar{\Omega}_{1} \subset \Omega_{2}$. Let the operator $T: P \cap\left(\bar{\Omega}_{2} \backslash \Omega_{1}\right) \rightarrow P$ be completely continuous, where $P$ is a cone in E. Suppose that one of the two conditions

(i) $\|T x\| \leq\|x\|, \forall x \in P \cap \partial \Omega_{1}$ and $\|T x\| \geq\|x\|, \forall x \in P \cap \partial \Omega_{2}$, and

(ii) $\|T x\| \geq\|x\|, \forall x \in P \cap \partial \Omega_{1}$, and $\|T x\| \leq\|x\|, \forall x \in P \cap \partial \Omega_{2}$, is satisfied. Then $T$ has at least one fixed point in $P \cap\left(\bar{\Omega}_{2} \backslash \Omega_{1}\right)$. 


\section{Main results}

In this part, applying Lemma 2.4, we get the optimal intervals of positive parameters $\lambda_{i}$ $(i=1,2,3)$ in which problem (1.1) admits at least two positive solutions.

Theorem 3.1 Assume that $\left(H_{1}\right)-\left(H_{8}\right)$ hold. Then we have:

(I) If $\alpha>1$, then there exist $\lambda_{1}^{*}, \lambda_{3}^{*}, \lambda^{*}, \lambda_{*}>0$ such that, for $\lambda_{i} \in\left[\lambda_{i}^{*}, \infty\right)(i=1,3)$ and $\lambda_{2} \in\left[\lambda_{*}, \lambda^{*}\right]$, problem (1.1) admits at least two positive solutions.

(II) If $0<\alpha<1$, then there exist $\lambda_{1}^{* *}, \lambda_{2}^{* *}, \lambda_{3}^{* *}>0$ such that, for $\lambda_{i} \in\left(0, \lambda_{i}^{*}\right]$, problem (1.1) admits at least two positive solutions.

Proof Part (I). Considering the case $\alpha>1$, it follows from $\left(H_{7}\right)$ that

$$
\lim _{x \rightarrow 0} \frac{f(x)}{x} \leq \lim _{x \rightarrow 0} \frac{k_{2} x^{\alpha}}{x}=0, \quad \lim _{x \rightarrow 0} \frac{I_{k}(x)}{x} \leq \lim _{x \rightarrow 0} \frac{l_{2} x^{\alpha}}{x}=0,
$$

which shows that there exists $r^{\prime}>0$ such that

$$
f(x) \leq \varepsilon_{1} x, \quad I_{k}(x) \leq \varepsilon_{2} x, \quad k=1,2, \ldots, n, x \in\left[0, r^{\prime}\right],
$$

where $\varepsilon_{1}, \varepsilon_{2}$ satisfy

$$
3 \lambda_{1} \varepsilon_{1} \int_{0}^{\xi} g^{+}(s) d s<1, \quad 3 n \lambda_{3} \varepsilon_{2}<1
$$

Let

$$
\begin{aligned}
& A=\lambda_{2} \int_{0}^{\xi} \int_{0}^{\xi} G_{1}(s, \tau) a^{+}(s) b^{+}(\tau) d \tau d s, \\
& A^{*}=\int_{0}^{\xi} \int_{0}^{\xi} G_{1}(s, \tau) a^{+}(s) b^{+}(\tau) d \tau d s,
\end{aligned}
$$

and choose $r=\min \left\{(3 A)^{-1}, \frac{r^{\prime}}{2}\right\}$. This implies $\lambda_{2} \leq \frac{1}{3 A^{*} r}$.

Then, for any $x \in K \cap \partial \Omega_{r}$, noticing (2.6), we get

$$
\begin{aligned}
(T x)(t)= & \lambda_{2} \int_{0}^{1} \int_{0}^{1} G(t, s) G_{1}(s, \tau) a(s) b(\tau) x(s) x(\tau) d \tau d s \\
& +\lambda_{1} \int_{0}^{1} G(t, s) g(s) f(x(s)) d s+\lambda_{3} \sum_{k=1}^{n} G_{s}^{\prime}\left(t, t_{k}\right) I_{k}\left(x\left(t_{k}\right)\right) \\
= & \lambda_{2} \int_{0}^{\xi} \int_{0}^{1} G(t, s) G_{1}(s, \tau) a^{+}(s) b(\tau) x(s) x(\tau) d \tau d s \\
& -\lambda_{2} \int_{\xi}^{1} \int_{0}^{1} G(t, s) G_{1}(s, \tau) a^{-}(s) b(\tau) x(s) x(\tau) d \tau d s \\
& +\lambda_{1} \int_{0}^{\xi} G(t, s) g^{+}(s) f(x(s)) d s-\lambda_{1} \int_{\xi}^{1} G(t, s) g^{-}(s) f(x(s)) d s \\
& +\lambda_{3} \sum_{k=1}^{n} G_{s}^{\prime}\left(t, t_{k}\right) I_{k}\left(x\left(t_{k}\right)\right)
\end{aligned}
$$




$$
\begin{aligned}
\leq & \lambda_{2} \int_{0}^{\xi} \int_{0}^{1} G(t, s) G_{1}(s, \tau) a^{+}(s) b(\tau) x(s) x(\tau) d \tau d s \\
& +\lambda_{1} \int_{0}^{\xi} G(t, s) g^{+}(s) f(x(s)) d s+\lambda_{3} \sum_{k=1}^{n} G_{s}^{\prime}\left(t, t_{k}\right) I_{k}\left(x\left(t_{k}\right)\right) \\
= & \lambda_{2} \int_{0}^{\xi} \int_{0}^{\xi} G(t, s) G_{1}(s, \tau) a^{+}(s) b^{+}(\tau) x(s) x(\tau) d \tau d s \\
& -\lambda_{2} \int_{0}^{\xi} \int_{0}^{\xi} G(t, s) G_{1}(s, \tau) a^{+}(s) b^{-}(\tau) x(s) x(\tau) d \tau d s \\
& +\lambda_{1} \int_{0}^{\xi} G(t, s) g^{+}(s) f(x(s)) d s+\lambda \sum_{k=1}^{n} G_{s}^{\prime}\left(t, t_{k}\right) I_{k}\left(x\left(t_{k}\right)\right) \\
< & \frac{1}{3}\|x\|_{P C}+\frac{1}{3}\|x\|_{P C}+\frac{1}{3}\|x\|_{P C} \\
= & \|x\|_{P C}, \int_{0}^{\xi} \int_{0}^{\xi} G(s, s) G_{1}(s, \tau) a^{+}(s) b^{+}(\tau) x(s) x(\tau) d \tau d s \\
& +\lambda_{2} \int_{0}^{\xi} \int_{0}^{\xi} G_{2}\|x\|_{P C}(s, \tau) a^{+}(s) b^{+}(\tau) x(s) x(\tau) d \tau d s+\lambda_{1} \int_{0}^{\xi} g^{+}(s) \varepsilon_{1} x(s) d s \\
& +\lambda_{1} \int_{0}^{\xi} G(s, s) g^{+}(s) f(x(s)) d s+\lambda \sum_{k=1}^{n} I_{k}\left(x\left(t_{k}\right)\right) \\
& +\lambda_{3} \sum_{k=1}^{n} I_{k}\left(x\left(t_{k}\right)\right) \\
\leq & \lambda_{2} \xi \int_{0}^{\xi} \int_{0}^{\xi} G_{1}(s, \tau) a^{+}(s) b^{+}(\tau) x(s) x(\tau) d \tau d s+\lambda_{1} \xi \int_{0}^{\xi} g^{+}(s) f(x(s)) d s
\end{aligned}
$$

which shows that

$$
\|T x\|_{P C}<\|x\|_{P C}, \quad \forall x \in K \cap \partial \Omega_{r} .
$$

On the other hand, noticing that $\alpha>1$, then it follows from $\left(H_{7}\right)$ that

$$
\lim _{x \rightarrow \infty} \frac{f(x)}{x} \geq \lim _{x \rightarrow \infty} \frac{k_{1} x^{\alpha}}{x}=\infty, \quad \lim _{x \rightarrow \infty} \frac{I_{k}(x)}{x} \geq \lim _{x \rightarrow \infty} \frac{l_{1} x^{\alpha}}{x}=\infty
$$

which shows that there exists $R^{\prime}>0$ such that

$$
f(x) \geq \varepsilon_{3} x, \quad I_{k}(x) \geq \varepsilon_{4} x, \quad x \geq R^{\prime},
$$


where $\varepsilon_{3}, \varepsilon_{4}$ satisfy

$$
\begin{aligned}
& \frac{3 \sigma_{2}}{2} \lambda_{1} \varepsilon_{3} \Gamma \int_{\frac{\sigma_{3}}{2}}^{\sigma_{3}} G(s, s) g^{+}(s) d s>1, \quad 3 \lambda_{3} \varepsilon_{4} \Gamma>1, \\
& \Gamma=\min _{\frac{\sigma_{3}}{2} \leq t \leq \sigma_{3}} \delta(t)>0, \quad \delta(t)=\min \left\{\frac{t}{\xi}, \frac{\xi-t}{\xi}\right\}, \quad t \in[0, \xi] .
\end{aligned}
$$

If $x \in K$, then from the concavity on $[0, \xi]$, it follows that

$$
x(t) \geq \delta(t)\|x\|_{P C}, \quad t \in[0, \xi] .
$$

Let $B$ and $B^{*}$ denote the following constants:

$$
\begin{aligned}
& B=\lambda_{2} \frac{\sigma_{2}}{2} \min _{\frac{\sigma_{2}}{2} \leq t \leq \sigma_{2}} \delta(t) \min _{\frac{\sigma_{1}}{2} \leq t \leq \sigma_{1}} \delta(t) \int_{\frac{\sigma_{2}}{2}}^{\sigma_{2}} \int_{\frac{\sigma_{1}}{2}}^{\sigma_{1}} G(s, s) G_{1}(s, \tau) a^{+}(s) b^{+}(\tau) d \tau d s>0, \\
& B^{*}=\frac{\sigma_{2}}{2} \min _{\frac{\sigma_{2}}{2} \leq t \leq \sigma_{2}} \delta(t) \min _{\frac{\sigma_{1}}{2} \leq t \leq \sigma_{1}} \delta(t) \int_{\frac{\sigma_{2}}{2}}^{\sigma_{2}} \int_{\frac{\sigma_{1}}{2}}^{\sigma_{1}} G(s, s) G_{1}(s, \tau) a^{+}(s) b^{+}(\tau) d \tau d s>0 .
\end{aligned}
$$

Noticing that, for all $d \in(0, \xi), \min _{\frac{d}{2} \leq t \leq d} \delta(t)=\min \left\{\frac{d}{2 \xi}, \frac{\xi-d}{\xi}\right\}>0, B$ and $B^{*}$ are defined well.

Setting $R=\max \left\{(3 B)^{-1}, \frac{R^{\prime}}{\Gamma}, r^{\prime}\right\}+1$, it follows that $\lambda_{2}>\lambda_{2}^{\prime}=\frac{1}{3 B^{*} R}$, and then for any $x \in$ $K \cap \partial \Omega_{R}$, we get

$$
\min _{\frac{\sigma_{3}}{2} \leq t \leq \sigma_{3}} x(t) \geq \min _{\frac{\sigma_{3}}{2} \leq t \leq \sigma_{3}} \delta(t)\|x\|_{P C} \geq \Gamma R>R^{\prime} .
$$

Then it follows from (2.6), (2.9), (3.8), and (3.9) that

$$
\begin{aligned}
\|T x\|_{P C}= & \max _{t \in J}\left\{\lambda_{2} \int_{0}^{1} \int_{0}^{1} G(t, s) G_{1}(s, \tau) a(s) b(\tau) x(s) x(\tau) d \tau d s\right. \\
& \left.+\lambda_{1} \int_{0}^{1} G(t, s) g(s) f(x(s)) d s+\lambda_{3} \sum_{k=1}^{n} G_{s}^{\prime}\left(t, t_{k}\right) I_{k}\left(x\left(t_{k}\right)\right)\right\} \\
\geq & \max _{t \in J}\left\{\lambda_{2} \int_{0}^{\sigma_{2}} G(t, s) a^{+}(s) x(s) \int_{0}^{1} G_{1}(s, \tau) b(\tau) x(\tau) d \tau d s\right. \\
& \left.+\lambda_{1} \int_{0}^{\sigma_{3}} G(t, s) g^{+}(s) f(x(s)) d s+\lambda_{3} \sum_{k=1}^{n} G_{s}^{\prime}\left(t, t_{k}\right) I_{k}\left(x\left(t_{k}\right)\right)\right\} \\
\geq & \lambda_{2} \min _{\frac{\sigma_{2}}{2} \leq t \leq \sigma_{2}} \int_{\frac{\sigma_{2}}{2}}^{\sigma_{2}} G(t, s) a^{+}(s) x(s) \int_{\frac{\sigma_{1}}{2}}^{\sigma_{1}} G_{1}(s, \tau) b^{+}(\tau) x(\tau) d \tau d s \\
& +\min _{\frac{\sigma_{2}}{2} \leq t \leq \sigma_{2}} \lambda_{1} \int_{\frac{\sigma_{3}}{2}}^{\sigma_{3}} G(t, s) g^{+}(s) f(x(s)) d s+\lambda_{3} \min _{\sigma_{3} \leq t \leq \xi} \sum_{\frac{t}{2}<t_{k}<t} I_{k}\left(x\left(t_{k}\right)\right) \\
\geq & \lambda_{2} \frac{\sigma_{2}}{2} \int_{\frac{\sigma_{2}}{2}}^{\sigma_{2}} G(s, s) a^{+}(s) \delta(s)\|x\|_{P C} \int_{\frac{\sigma_{1}}{2}}^{\sigma_{1}} G_{1}(s, \tau) b^{+}(\tau) \delta(\tau)\|x\|_{P C} d \tau d s \\
& +\lambda_{1} \frac{\sigma_{2}}{2} \int_{\frac{\sigma_{3}}{2}}^{\sigma_{3}} G(s, s) g^{+}(s) \varepsilon_{3} x(s) d s+\lambda_{3} \sum_{\frac{\sigma_{3}}{2}<t_{k}<\sigma_{3}} \varepsilon_{4} x\left(t_{k}\right)
\end{aligned}
$$




$$
\begin{aligned}
\geq & \lambda_{2} \frac{\sigma_{2}}{2} \int_{\frac{\sigma_{2}}{2}}^{\sigma_{2}} G(s, s) a^{+}(s) \delta(s)\|x\|_{P C} \int_{\frac{\sigma_{1}}{2}}^{\sigma_{1}} G_{1}(s, \tau) b^{+}(\tau) \delta(\tau)\|x\|_{P C} d \tau d s \\
& +\lambda_{1} \frac{\sigma_{2}}{2} \int_{\frac{\sigma_{3}}{2}}^{\sigma_{3}} G(s, s) g^{+}(s) \varepsilon_{3} x(s) d s+\lambda_{3} \varepsilon_{4} \sum_{\frac{\sigma_{3}}{2}<t_{1}<\sigma_{3}} x\left(t_{1}\right) \\
\geq & B\|x\|_{P C}^{2}+\lambda_{1} \frac{\sigma_{2}}{2} \varepsilon_{3} \min _{\frac{\sigma_{3}}{2} \leq t \leq \sigma_{3}} \delta(t) \int_{\frac{\sigma_{3}}{2}}^{\sigma_{3}} G(s, s) g^{+}(s) d s\|x\|_{P C} \\
& +\lambda_{3} \varepsilon_{4} \min _{\frac{\sigma_{3}}{2} \leq t \leq \sigma_{3}} \delta(t)\|x\|_{P C} \\
> & \frac{1}{3}\|x\|_{P C}+\frac{1}{3}\|x\|_{P C}+\frac{1}{3}\|x\|_{P C} \\
= & \|x\|_{P C} .
\end{aligned}
$$

\section{Consequently,}

$$
\|T x\|_{P C}>\|x\|_{P C}, \quad x \in K \cap \partial \Omega_{R} .
$$

Let

$$
\lambda_{1}^{*}=\frac{\left(6 D A^{*}-2 B^{*}\right) \eta}{3 D A^{*} \sigma_{2} \sigma_{\eta} \int_{\frac{\sigma_{3}}{2}}^{\sigma_{3}} G(s, s) g^{+}(s) d s}, \quad \lambda_{2}^{*}=\frac{\frac{1}{2}}{3 D A^{*} \eta}, \quad \lambda_{3}^{*}=\frac{\frac{B^{*}}{2} \eta}{3 D A^{*} \sigma_{\eta}^{*}}
$$

where $0<\eta<r, D=\frac{r^{2}}{\eta^{2}}, A^{*}$ is defined in (3.3), and $B^{*}$ is defined in (3.9).

We define $\sigma_{\eta}$ and $\sigma_{\eta}^{*}$ by

$$
\begin{aligned}
& \sigma_{\eta}=\min _{\Gamma \eta \leq x \leq \eta}\{f(x)\}>0, \quad \sigma_{\eta}^{*}=\min \left\{m_{k}, k=1,2, \ldots, n\right\}>0, \\
& m_{k}=\min _{\Gamma \eta \leq x \leq \eta}\left\{I_{k}(x)\right\}, \quad k=1,2, \ldots, n,
\end{aligned}
$$

where $\Gamma$ is defined in (3.6). Noticing $\left(H_{2}\right)$ and $\left(H_{3}\right)$, then $\sigma_{\eta}$ and $\sigma_{\eta}^{*}$ are defined well and $\sigma_{\eta}>0, \sigma_{\eta}^{*}>0$. So, for any $x \in K \cap \partial \Omega_{\eta}$, we get

$$
\Gamma \eta \leq \Gamma\|x\|_{P C} \leq \min _{\frac{\sigma_{3}}{2} \leq t \leq \sigma_{3}} x(t) \leq x(t) \leq \eta, \quad \frac{\sigma_{3}}{2} \leq t \leq \sigma_{3},
$$

and hence, it follows from (2.6), (2.7), (3.7), and (3.9) that

$$
\begin{aligned}
\|T x\|_{P C}= & \max _{t \in J}\left\{\lambda_{2} \int_{0}^{1} \int_{0}^{1} G(t, s) G_{1}(s, \tau) a(s) b(\tau) x(s) x(\tau) d \tau d s\right. \\
& \left.+\lambda_{1} \int_{0}^{1} G(t, s) g(s) f(x(s)) d s+\lambda_{3} \sum_{k=1}^{n} G_{s}^{\prime}\left(t, t_{k}\right) I_{k}\left(x\left(t_{k}\right)\right)\right\} \\
\geq & \max _{t \in J}\left\{\lambda_{2} \int_{0}^{\sigma_{2}} G(t, s) a^{+}(s) x(s) \int_{0}^{1} G_{1}(s, \tau) b(\tau) x(\tau) d \tau d s\right. \\
& \left.+\lambda_{1} \int_{0}^{\sigma_{3}} G(t, s) g^{+}(s) f(x(s)) d s+\lambda_{3} \sum_{k=1}^{n} G_{s}^{\prime}\left(t, t_{k}\right) I_{k}\left(x\left(t_{k}\right)\right)\right\}
\end{aligned}
$$




$$
\begin{aligned}
& \geq \lambda_{2} \min _{\frac{\sigma_{2}}{2} \leq t \leq \sigma_{2}} \int_{\frac{\sigma_{2}}{2}}^{\sigma_{2}} G(t, s) a^{+}(s) x(s) \int_{\frac{\sigma_{1}}{2}}^{\sigma_{1}} G_{1}(s, \tau) b^{+}(\tau) x(\tau) d \tau d s \\
& +\min _{\frac{\sigma_{2}}{2} \leq t \leq \sigma_{2}} \lambda_{1} \int_{\frac{\sigma_{3}}{2}}^{\sigma_{3}} G(t, s) g^{+}(s) f(x(s)) d s+\lambda_{3} \min _{\sigma_{3} \leq t \leq \xi} \sum_{\frac{t}{2}<t_{k}<t} I_{k}\left(x\left(t_{k}\right)\right) \\
& \geq \lambda_{2} \frac{\sigma_{2}}{2} \int_{\frac{\sigma_{2}}{2}}^{\sigma_{2}} G(s, s) a^{+}(s) \delta(s)\|x\|_{P C} \int_{\frac{\sigma_{1}}{2}}^{\sigma_{1}} G_{1}(s, \tau) b^{+}(\tau) \delta(\tau)\|x\|_{P C} d \tau d s \\
& +\lambda_{1} \frac{\sigma_{2}}{2} \int_{\frac{\sigma_{3}}{2}}^{\sigma_{3}} G(s, s) g^{+}(s) \sigma_{\eta} d s+\lambda_{3} \sum_{\frac{\sigma_{3}}{2}<t_{k}<\sigma_{3}} \sigma_{\eta}^{*} \\
& \geq \lambda_{2} \frac{\sigma_{2}}{2} \int_{\frac{\sigma_{2}}{2}}^{\sigma_{2}} G(s, s) a^{+}(s) \delta(s)\|x\|_{P C} \int_{\frac{\sigma_{1}}{2}}^{\sigma_{1}} G_{1}(s, \tau) b^{+}(\tau) \delta(\tau)\|x\|_{P C} d \tau d s \\
& +\lambda_{1} \frac{\sigma_{2}}{2} \int_{\frac{\sigma_{3}}{2}}^{\sigma_{3}} G(s, s) g^{+}(s) \sigma_{\eta} d s+\lambda_{3} \sigma_{\eta}^{*} \\
& \geq \lambda_{2}^{*} B^{*}\|x\|_{P C}^{2}+\lambda_{1}^{*} \frac{\sigma_{2}}{2} \sigma_{\eta} \int_{\frac{\sigma_{3}}{2}}^{\sigma_{3}} G(s, s) g^{+}(s) d s+\lambda_{3}^{*} \sigma_{\eta}^{*} \\
& =\frac{\frac{B^{*}}{2}}{3 D A^{*}}\|x\|_{P C}+\frac{3 D A^{*}-B^{*}}{3 D A^{*}}\|x\|_{P C}+\frac{\frac{B^{*}}{2}}{3 D A^{*}}\|x\|_{P C} \\
& =\|x\|_{P C} \text {. }
\end{aligned}
$$

This shows

$$
\|T x\|_{P C}>\|x\|_{P C}, \quad x \in K \cap \partial \Omega_{\eta} .
$$

Let $\lambda_{*}=\max \left\{\lambda_{2}^{\prime}, \lambda_{2}^{*}\right\}, \lambda^{*}=\frac{1}{3 A^{*} r}$, then, for $\lambda_{i} \in\left[\lambda_{i}^{*},+\infty\right), i=1,3$, and $\lambda_{2} \in\left[\lambda_{*}, \lambda^{*}\right]$, applying Lemma 2.4 to (3.5), (3.10), and (3.11) yields that $T$ has two fixed points $x_{1}$ and $x_{2}$ satisfying $x_{1} \in K \cap\left(\bar{\Omega}_{R} \backslash \bar{\Omega}_{r}\right), x_{2} \in K \cap\left(\Omega_{r} \backslash \Omega_{\eta}\right)$. Thus it follows from Remark 2.3 that problem (1.1) admits at least two positive solutions $\left(x_{i}, y_{i}\right)(i=1,2)$ with $x_{1} \in K \cap\left(\bar{\Omega}_{R} \backslash \bar{\Omega}_{r}\right), x_{2} \in$ $K \cap\left(\Omega_{r} \backslash \Omega_{\eta}\right)$, and

$$
\begin{aligned}
& y_{1}(t)=\lambda_{2} \int_{0}^{1} G_{1}(t, s) b(s) x_{1}(s) d s, \\
& y_{2}(t)=\lambda_{2} \int_{0}^{1} G_{1}(t, s) b(s) x_{2}(s) d s .
\end{aligned}
$$

Part (II). Next turning to $0<\alpha<1$, it follows from $\left(H_{7}\right)$ that

$$
\begin{aligned}
& \lim _{x \rightarrow 0} \frac{f(x)}{x} \geq \lim _{x \rightarrow 0} \frac{k_{1} x^{\alpha}}{x}=+\infty, \\
& \lim _{x \rightarrow 0} \frac{I_{k}(x)}{x} \geq \lim _{x \rightarrow 0} \frac{l_{1} x^{\alpha}}{x}=+\infty,
\end{aligned}
$$

which shows that there exists a positive constant $r>0$ such that

$$
f(x) \geq \varepsilon_{5} x, \quad I_{k}(x) \geq \varepsilon_{6} x, \quad k=1,2, \ldots, n, 0 \leq x \leq r,
$$


where $\varepsilon_{5}, \varepsilon_{6}$ satisfy

$$
\lambda_{1} \sigma_{2} \varepsilon_{5} \min _{\frac{\sigma_{3}}{2} \leq t \leq \sigma_{3}} \delta(t) \int_{\frac{\sigma_{3}}{2}}^{\sigma_{3}} G(s, s) g^{+}(s) d s>1, \quad 2 \lambda_{3} \varepsilon_{6} \min _{\frac{\sigma_{3}}{2} \leq t \leq \sigma_{3}} \delta(t)>1,
$$

where $\delta(t)$ is defined in (3.6), and $A$ is defined in (3.2).

Therefore, for any $x \in K \cap \partial \Omega_{r}$, noticing (3.7), we get

$$
\begin{aligned}
& \|T x\|_{P C}=\max _{t \in J}\left\{\lambda_{2} \int_{0}^{1} \int_{0}^{1} G(t, s) G_{1}(s, \tau) a(s) b(\tau) x(s) x(\tau) d \tau d s\right. \\
& \left.+\lambda_{1} \int_{0}^{1} G(t, s) g(s) f(x(s)) d s+\lambda_{3} \sum_{k=1}^{n} G_{s}^{\prime}\left(t, t_{k}\right) I_{k}\left(x\left(t_{k}\right)\right)\right\} \\
& \geq \max _{t \in J}\left\{\lambda_{2} \int_{0}^{\sigma_{2}} G(t, s) a^{+}(s) x(s) \int_{0}^{1} G_{1}(s, \tau) b(\tau) x(\tau) d \tau d s\right. \\
& \left.+\lambda_{1} \int_{0}^{\sigma_{3}} G(t, s) g^{+}(s) f(x(s)) d s+\lambda_{3} \sum_{k=1}^{n} G_{s}^{\prime}\left(t, t_{k}\right) I_{k}\left(x\left(t_{k}\right)\right)\right\} \\
& \geq \lambda_{2} \min _{\frac{\sigma_{2}}{2} \leq t \leq \sigma_{2}} \int_{\frac{\sigma_{2}}{2}}^{\sigma_{2}} G(t, s) a^{+}(s) x(s) \int_{\frac{\sigma_{1}}{2}}^{\sigma_{1}} G_{1}(s, \tau) b^{+}(\tau) x(\tau) d \tau d s \\
& +\min _{\frac{\sigma_{2}}{2} \leq t \leq \sigma_{2}} \lambda_{1} \int_{\frac{\sigma_{3}}{2}}^{\sigma_{3}} G(t, s) g^{+}(s) f(x(s)) d s+\lambda_{3} \min _{\sigma_{3} \leq t \leq \xi} \sum_{\frac{t}{2}<t_{k}<t} I_{k}\left(x\left(t_{k}\right)\right) \\
& \geq \lambda_{2} \frac{\sigma_{2}}{2} \int_{\frac{\sigma_{2}}{2}}^{\sigma_{2}} G(s, s) a^{+}(s) \delta(s)\|x\|_{P C} \int_{\frac{\sigma_{1}}{2}}^{\sigma_{1}} G_{1}(s, \tau) b^{+}(\tau) \delta(\tau)\|x\|_{P C} d \tau d s \\
& +\lambda_{1} \frac{\sigma_{2}}{2} \int_{\frac{\sigma_{3}}{2}}^{\sigma_{3}} G(s, s) g^{+}(s) \varepsilon_{5} x(s) d s+\lambda_{3} \sum_{\frac{\sigma_{3}}{2}<t_{k}<\sigma_{3}} \varepsilon_{6} x\left(t_{k}\right) \\
& \geq \lambda_{1} \frac{\sigma_{2}}{2} \int_{\frac{\sigma_{3}}{2}}^{\sigma_{3}} G(s, s) g^{+}(s) \varepsilon_{5} x(s) d s+\lambda_{3} \varepsilon_{6} \sum_{\frac{\sigma_{3}}{2}<t_{1}<\sigma_{3}} x\left(t_{1}\right) \\
& \geq \lambda_{1} \frac{\sigma_{2}}{2} \varepsilon_{5} \min _{\frac{\sigma_{3}}{2} \leq t \leq \sigma_{3}} \delta(t) \int_{\frac{\sigma_{3}}{2}}^{\sigma_{3}} G(s, s) g^{+}(s) d s\|x\|_{P C}+\lambda_{3} \varepsilon_{6} \min _{\frac{\sigma_{3}}{2} \leq t \leq \sigma_{3}} \delta(t)\|x\|_{P C} \\
& >\frac{1}{2}\|x\|_{P C}+\frac{1}{2}\|x\|_{P C} \\
& =\|x\|_{P C} \text {. }
\end{aligned}
$$

Consequently,

$$
\|T x\|_{P C}>\|x\|_{P C}, \quad x \in K \cap \partial \Omega_{r} .
$$

On the other hand, notice that $0<\alpha<1$, then it follows from $\left(H_{7}\right)$ that

$$
\lim _{x \rightarrow \infty} \frac{f(x)}{x} \leq \lim _{x \rightarrow \infty} \frac{k_{2} x^{\alpha}}{x}=0, \quad \lim _{x \rightarrow \infty} \frac{I_{k}(x)}{x} \leq \lim _{x \rightarrow \infty} \frac{l_{2} x^{\alpha}}{x}=0,
$$


which shows that there exists $R^{\prime}>r$ such that

$$
f(x) \leq \varepsilon_{7} x, \quad I_{k}(x) \leq \varepsilon_{8} x, \quad x \geq R^{\prime}
$$

where $\varepsilon_{7}, \varepsilon_{8}$ satisfy

$$
5 \lambda_{1} \varepsilon_{7} \int_{D(x)} g^{+}(s) d s<1, \quad 5 \lambda_{3} n \varepsilon_{8}<1
$$

Let

$$
M=\lambda_{1} \max _{\|x\|_{P C}=R^{\prime}} f(x) \int_{0}^{\xi} g^{+}(s) d s, \quad M^{\prime}=\lambda_{1} n \max _{\|x\|_{P C}=R^{\prime}} I_{k}(x) .
$$

Then it is not difficult to see that $M<+\infty$ and $M^{\prime}<+\infty$.

Choosing $\max \left\{R^{\prime}, 5 M, 5 M^{\prime}\right\}<R<(5 A)^{-1}$, then $M<\frac{R}{5}, M^{\prime}<\frac{R}{5}$.

Now, choosing $x \in K \cap \partial \Omega_{R}$ arbitrarily and letting $\bar{x}(t)=\min \left\{x(t), R^{\prime}\right\}$, then $\bar{x}(t) \leq R^{\prime}$. Moreover, write $D(x)=\left\{t \in[0, \xi]: x(t)>R^{\prime}\right\}$. Therefore, for $t \in D(x)$, we get $R^{\prime}<x(t) \leq$ $\|x\|_{P C}=R, \forall t \in D(x)$. By the choice of $R^{\prime}$, for $t \in D(x)$, we have $f(x) \leq \varepsilon_{1} R$.

Similarly, one can define $D^{\prime}(x)=\left\{t_{k} \in J: x\left(t_{k}\right)>R^{\prime}\right\}$. Then, for $t_{k} \in D^{\prime}(x)$, we get $R^{\prime}<$ $x\left(t_{k}\right) \leq\|x\|_{P C}=R, \forall t \in D^{\prime}(x)$. By the choice of $R^{\prime}$, for $t_{k} \in D^{\prime}(x)$, we get $I_{k}\left(x\left(t_{k}\right)\right) \leq \varepsilon_{2} R$, $k=1,2, \ldots, n$.

Therefore, for any $x \in K \cap \partial \Omega_{R}$, we get

$$
\begin{aligned}
(T x)(t)= & \lambda_{2} \int_{0}^{1} \int_{0}^{1} G(t, s) G_{1}(s, \tau) a(s) b(\tau) x(s) x(\tau) d \tau d s \\
& +\lambda_{1} \int_{0}^{1} G(t, s) g(s) f(x(s)) d s+\lambda_{3} \sum_{k=1}^{n} G_{s}^{\prime}\left(t, t_{k}\right) I_{k}\left(x\left(t_{k}\right)\right) \\
= & \lambda_{2} \int_{0}^{\xi} \int_{0}^{1} G(t, s) G_{1}(s, \tau) a^{+}(s) b(\tau) x(s) x(\tau) d \tau d s \\
& -\lambda_{2} \int_{\xi}^{1} \int_{0}^{1} G(t, s) G_{1}(s, \tau) a^{-}(s) b(\tau) x(s) x(\tau) d \tau d s \\
& +\lambda_{1} \int_{0}^{\xi} G(t, s) g^{+}(s) f(x(s)) d s-\lambda_{1} \int_{\xi}^{1} G(t, s) g^{-}(s) f(x(s)) d s \\
& +\lambda_{3} \sum_{k=1}^{n} G_{s}^{\prime}\left(t, t_{k}\right) I_{k}\left(x\left(t_{k}\right)\right) \\
\leq & \lambda_{2} \int_{0}^{\xi} \int_{0}^{1} G(t, s) G_{1}(s, \tau) a^{+}(s) b(\tau) x(s) x(\tau) d \tau d s \\
& +\lambda_{1} \int_{0}^{\xi} G(t, s) g^{+}(s) f(x(s)) d s+\lambda_{3} \sum_{k=1}^{n} G_{s}^{\prime}\left(t, t_{k}\right) I_{k}\left(x\left(t_{k}\right)\right) \\
= & \lambda_{2} \int_{0}^{\xi} \int_{0}^{\xi} G(t, s) G_{1}(s, \tau) a^{+}(s) b^{+}(\tau) x(s) x(\tau) d \tau d s \\
& -\lambda_{2} \int_{0}^{\xi} \int_{0}^{\xi} G(t, s) G_{1}(s, \tau) a^{+}(s) b^{-}(\tau) x(s) x(\tau) d \tau d s
\end{aligned}
$$




$$
\begin{aligned}
& +\lambda_{1} \int_{0}^{\xi} G(t, s) g^{+}(s) f(x(s)) d s+\lambda_{3} \sum_{k=1}^{n} G_{s}^{\prime}\left(t, t_{k}\right) I_{k}\left(x\left(t_{k}\right)\right) \\
& \leq \lambda_{2} \int_{0}^{\xi} \int_{0}^{\xi} G(s, s) G_{1}(s, \tau) a^{+}(s) b^{+}(\tau) x(s) x(\tau) d \tau d s \\
& +\lambda_{1} \int_{0}^{\xi} G(s, s) g^{+}(s) f(x(s)) d s+\lambda_{3} \sum_{k=1}^{n} I_{k}\left(x\left(t_{k}\right)\right) \\
& \leq \lambda_{2} \xi \int_{0}^{\xi} \int_{0}^{\xi} G_{1}(s, \tau) a^{+}(s) b^{+}(\tau) x(s) x(\tau) d \tau d s+\lambda_{1} \xi \int_{0}^{\xi} g^{+}(s) f(x(s)) d s \\
& +\lambda_{3} \sum_{k=1}^{n} I_{k}\left(x\left(t_{k}\right)\right) \\
& <\lambda_{2} \int_{0}^{\xi} \int_{0}^{\xi} G_{1}(s, \tau) a^{+}(s) b^{+}(\tau) x(s) x(\tau) d \tau d s+\lambda_{1} \int_{D(x)} g^{+}(s) f(x(s)) d s \\
& +\lambda_{1} \int_{[0, \xi] \backslash D(x)} g^{+}(s) f(x(s)) d s+\lambda_{3} \sum_{t_{k} \in D^{\prime}(x)} I_{k}\left(x\left(t_{k}\right)\right) \\
& +\lambda_{3} \sum_{t_{k} \in J \backslash D^{\prime}(x)} I_{k}\left(x\left(t_{k}\right)\right) \\
& \leq \lambda_{2} \int_{0}^{\xi} \int_{0}^{\xi} G_{1}(s, \tau) a^{+}(s) b^{+}(\tau) x(s) x(\tau) d \tau d s+\lambda_{1} \int_{D(x)} g^{+}(s) f(x(s)) d s \\
& +\lambda_{1} \int_{0}^{\xi} g^{+}(s) f(\bar{x}(s)) d s+\lambda_{3} \sum_{t_{k} \in D^{\prime}(x)} I_{k}\left(x\left(t_{k}\right)\right)+\lambda_{3} \sum_{k=1}^{n} I_{k}\left(\bar{x}\left(t_{k}\right)\right) \\
& \leq \lambda_{2} \int_{0}^{\xi} \int_{0}^{\xi} G_{1}(s, \tau) a^{+}(s) b^{+}(\tau) d \tau d s\|x\|_{P C}^{2}+\lambda_{1} \varepsilon_{7} \int_{D(x)} g^{+}(s) d s\|x\|_{P C} \\
& +M+\lambda_{3} n \varepsilon_{8}\|x\|_{P C}+M^{\prime} \\
& <\frac{1}{5}\|x\|_{P C}+\frac{1}{5}\|x\|_{P C}+\frac{1}{5}\|x\|_{P C}+\frac{1}{5}\|x\|_{P C}+\frac{1}{5}\|x\|_{P C} \\
& =\|x\|_{P C},
\end{aligned}
$$

which shows that

$$
\|T x\|_{P C}<\|x\|_{P C}, \quad \forall x \in K \cap \partial \Omega_{R} .
$$

Let

$$
\lambda_{1}^{* *}=\frac{\eta}{3 M_{\eta} \int_{0}^{\xi} g^{+}(s) d s}, \quad \lambda_{2}^{* *}=\frac{1}{3 A^{*} R}<\frac{1}{3 A^{*} \eta}, \quad \lambda_{3}^{* *}=\frac{\eta}{3 n M_{\eta}^{*}}
$$

where $0<\eta<r$, and $A^{*}$ is defined in (3.3).

We define $M_{\eta}$ and $M_{\eta}^{*}$ by

$$
\begin{aligned}
& M_{\eta}=\max _{0 \leq x \leq \eta}\{f(x)\}>0, \quad M_{\eta}^{*}=\max \left\{m_{k}, k=1,2, \ldots, n\right\}>0, \\
& m_{k}=\min _{0 \leq x \leq \eta}\left\{I_{k}(x)\right\}, \quad k=1,2, \ldots, n .
\end{aligned}
$$


Noticing $\left(H_{2}\right)$ and $\left(H_{3}\right)$, then $M_{\eta}$ and $M_{\eta}^{*}$ are defined well and $M_{\eta}>0, M_{\eta}^{*}>0$. So, for any $x \in K \cap \partial \Omega_{\eta}$, similar to the proof of (3.4), we get

$$
\begin{aligned}
\|T x\|_{P C} \leq & \lambda_{2} \int_{0}^{\xi} \int_{0}^{\xi} G(s, \tau) a^{+}(s) b^{+}(\tau) x(s) x(\tau) d \tau d s \\
& +\lambda_{1} \int_{0}^{\xi} g^{+}(s) f(x(s)) d s+\lambda_{3} \sum_{k=1}^{n} I_{k}\left(x\left(t_{k}\right)\right) \\
\leq & \lambda_{2}\|x\|_{P C}^{2} A^{*}+\lambda_{1} \int_{0}^{\xi} g^{+}(s) d s M_{\eta}+n \lambda_{3} M_{\eta}^{*} \\
\leq & \lambda_{2}^{* *}\|x\|_{P C}^{2} A^{*}+\lambda_{1}^{* *} \int_{0}^{\xi} g^{+}(s) d s M_{\eta}+n \lambda_{3}^{* *} M_{\eta}^{*} \\
\leq & \frac{1}{3}\|x\|_{P C}+\frac{1}{3}\|x\|_{P C}+\frac{1}{3}\|x\|_{P C} \\
= & \|x\|_{P C},
\end{aligned}
$$

which shows

$$
\|T x\|_{P C} \leq\|x\|_{P C}, \quad x \in K \cap \partial \Omega_{\eta} .
$$

Therefore, applying Lemma 2.4 to (3.12), (3.14), and (3.15) yields $T$ has two fixed points $x_{1}$ and $x_{2}$ satisfying $x_{1} \in K \cap\left(\bar{\Omega}_{R} \backslash \bar{\Omega}_{r}\right), x_{2} \in K \cap\left(\Omega_{r} \backslash \Omega_{\eta}\right)$. Thus it follows from Remark 2.3 that problem (1.1) admits at least two positive solutions $\left(x_{i}, y_{i}\right)(i=1,2)$ with $x_{1} \in K \cap\left(\bar{\Omega}_{R} \backslash\right.$ $\left.\bar{\Omega}_{r}\right), x_{2} \in K \cap\left(\Omega_{r} \backslash \Omega_{\eta}\right)$, and

$$
\begin{aligned}
& y_{1}(t)=\lambda_{2} \int_{0}^{1} G_{1}(t, s) b(s) x_{1}(s) d s, \\
& y_{2}(t)=\lambda_{2} \int_{0}^{1} G_{1}(t, s) b(s) x_{2}(s) d s .
\end{aligned}
$$

The proof of Theorem 3.1 is complete.

\section{Remarks and comments}

In this section, we offer some remarks and comments on the associated problem (1.1).

Remark 4.1 It is not difficult to see that the details of the proof of (3.4), (3.10) are very different from those of (3.12) and (3.13), respectively. And the idea to prove (3.13) comes from Theorem 3.2 of [50].

Remark 4.2 Some ideas of dealing with parameters in Theorem 3.1 come from Theorems 2.1-2.4 in [51], but there are very few papers in the literature considering the multiplicity of positive solutions for second-order impulsive differential system, especially under the case the weight functions $a(t), b(t)$, and $g(t)$ change sign on $[0,1]$.

Remark 4.3 It is not difficult to see that conditions $\left(H_{2}\right),\left(H_{3}\right)$, and $\left(H_{7}\right)$ play an important role in the proof of Theorem 3.1, and there are many functions satisfying $\left(H_{2}\right),\left(H_{3}\right)$, and 
$\left(H_{7}\right)$, for example,

$$
f(x)=x^{\alpha}, \quad I_{k}(x)=x^{\alpha}, \quad k=1,2, \ldots, n, 0<\alpha<+\infty .
$$

Remark 4.4 If we respectively replace conditions $\left(H_{2}\right)$ and $\left(H_{3}\right)$ by

$\left(H_{2}^{\prime}\right) f \in C\left(\mathcal{R}^{+}, \mathcal{R}^{+}\right)$,

$\left(H_{3}^{\prime}\right) I_{k} \in C\left(\mathcal{R}^{+}, \mathcal{R}^{+}\right)$,

then we cannot guarantee $\sigma_{\eta}>0, \sigma_{\eta}^{*}>0, M_{\eta}>$ and $M_{\eta}^{*}>0$, which are respectively defined in Theorem 3.1. Further, we cannot obtain the results of Theorem 3.1.

Remark 4.5 Similarly, one can consider the multiplicity of positive solutions for the following nonlocal impulsive systems:

$$
\begin{cases}-x^{\prime \prime}=a(t) x y+\lambda_{1} g(t) f(x), & 0<t<1, t \neq t_{k}, \\ -y^{\prime \prime}=\lambda_{2} b(t) x, & 0<t<1, \\ \left.\Delta x\right|_{t=t_{k}}=\lambda_{3} I_{k}\left(x\left(t_{k}\right)\right), & \\ \left.\Delta x^{\prime}\right|_{t=t_{k}}=0, & k=1,2, \ldots, n, \\ x(0)=0, & x^{\prime}(1)=0,\end{cases}
$$

where $\lambda_{1}>0, \lambda_{2}>0$, and $\lambda_{3}>0$ are three parameters, $a(t), b(t), g(t)$ change sign on $[0,1]$.

On the other hand, we conjecture that the conclusion in Theorem 3.1 holds for the following systems:

$$
\begin{cases}-x^{\prime \prime}=a(t) x y+\lambda_{1} g(t) f(x), & 0<t<1, t \neq t_{k}, \\ -y^{\prime \prime}=\lambda_{2} b(t) x, & 0<t<1, \\ -\left.\Delta x^{\prime}\right|_{t=t_{k}}=\lambda_{3} I_{k}\left(x\left(t_{k}\right)\right), & k=1,2, \ldots, n, \\ x(0)=0, \quad x^{\prime}(1)=0, & \\ y(0)=y(1)=0, & \end{cases}
$$

and

$$
\begin{cases}-x^{\prime \prime}=a(t) x y+\lambda_{1} g(t) f(x), & 0<t<1, t \neq t_{k}, \\ -y^{\prime \prime}=\lambda_{2} b(t) x, & 0<t<1, \\ -\left.\Delta x^{\prime}\right|_{t=t_{k}}=\lambda_{3} I_{k}\left(x\left(t_{k}\right)\right), & k=1,2, \ldots, n, \\ x(0)=0, \quad x^{\prime}(1)=0, & \\ y(0)=0, \quad y^{\prime}(1)=0, & \end{cases}
$$

where $\lambda_{1}>0, \lambda_{2}>0$, and $\lambda_{3}>0$ are three parameters, $a(t), b(t), g(t)$ change sign on $[0,1]$. 


\section{An example}

Example 5.1 Let $n=1, t_{1}=\frac{1}{5}$. Consider the following system:

$$
\begin{cases}-x^{\prime \prime}=a(t) x y+\lambda_{1} g(t) \sqrt{x}, & 0<t<1, t \neq \frac{1}{5}, \\ -y^{\prime \prime}=\lambda_{2} b(t) x, & 0<t<1, \\ \left.\Delta x\right|_{t=\frac{1}{5}}=\lambda_{3} I_{1}\left(x\left(\frac{1}{5}\right)\right), & \\ \left.\Delta x^{\prime}\right|_{t=\frac{1}{5}}=0, & \\ x(0)=0, \quad x^{\prime}(1)=0, & \\ y(0)=y(1)=0, & \end{cases}
$$

where $I_{1}(x)=\sqrt{x}$ and

$$
\begin{aligned}
& b(t)= \begin{cases}\frac{128}{3}\left(\frac{1}{3}-t\right), & t \in\left[0, \frac{1}{3}\right], \\
-\frac{1}{16}\left(t-\frac{1}{3}\right), & t \in\left[\frac{1}{3}, 1\right],\end{cases} \\
& a(t)= \begin{cases}\frac{2048}{3}\left(\frac{1}{3}-t\right), & t \in\left[0, \frac{1}{3}\right], \\
-\frac{1}{16}\left(t-\frac{1}{3}\right), & t \in\left[\frac{1}{3}, 1\right],\end{cases} \\
& g(t)= \begin{cases}\frac{64}{\sqrt{3}}\left(\frac{1}{3}-t\right), & t \in\left[0, \frac{1}{3}\right], \\
-\frac{1}{16}\left(t-\frac{1}{3}\right), & t \in\left[\frac{1}{3}, 1\right] .\end{cases}
\end{aligned}
$$

Firstly, we give a result which is similar to that of Proposition 2.3 in [36]. Consider the following boundary value problems:

$$
\begin{aligned}
& \left\{\begin{array}{l}
-x^{\prime \prime}=k(t) x^{\alpha}, \\
x(0)=0, \quad x^{\prime}(1)=0,
\end{array}\right. \\
& \left\{\begin{array}{l}
-x^{\prime \prime}=k(t) x^{\alpha}, \quad 0<t<1, \\
x(0)=x(1)=0,
\end{array}\right.
\end{aligned}
$$

where $\alpha>0$ with $\alpha \neq 1, k(t)$ satisfies the changing sign condition

$$
\begin{cases}k(t) \geq 0, & t \in[0, \xi], \\ k(t) \leq 0, & t \in[\xi, 1],\end{cases}
$$

and

$$
c_{1} x^{\alpha} \leq f(x)=x^{\alpha} \leq c_{2} x^{\alpha}, \quad c_{1}, c_{2}>0 .
$$

If there exists $0<\sigma<\xi$ such that

$$
c_{1} \frac{\xi-\sigma}{1-\xi} \sigma^{\alpha+1} \mu^{2} k^{+}\left(\xi-\frac{\xi-\sigma}{1-\xi} \tau\right) \geq c_{2} \xi^{\alpha} k^{-}(\xi+\tau), \quad \tau \in[0,1-\xi]
$$


then the following inequalities hold:

$$
\begin{aligned}
\sigma^{\alpha} \mu^{2} \int_{\sigma}^{\xi} G(t, s) k^{+}(s) d s & \geq \frac{c_{2}}{c_{1}} \xi^{\alpha} \int_{\xi}^{1} G(t, s) k^{-}(s) d s, \\
\sigma^{\alpha} \mu^{2} \int_{\sigma}^{\xi} G_{1}(t, s) k^{+}(s) d s & \geq \frac{c_{2}}{c_{1}} \xi^{\alpha} \int_{\xi}^{1} G_{1}(t, s) k^{-}(s) d s .
\end{aligned}
$$

Next, we study the multiplicity of positive solutions of problem (5.1). From definitions of $a(t), b(t)$, and $g(t)$, we know that $\xi=\frac{1}{3}$.

Step 1. We show that $\left(H_{4}\right)$ holds. For fixed $c_{1}=c_{2}=1, \sigma_{1}=\frac{1}{4}, \mu=1$, and $\alpha=1,(5.4)$ is equivalent to the following inequality:

$$
\frac{3}{128} b^{+}\left(\frac{1}{3}-\frac{1}{8} \tau\right) \geq b^{-}\left(\frac{1}{3}+\tau\right), \quad \tau \in\left[0, \frac{2}{3}\right] \text {. }
$$

Letting $\frac{1}{3}-\frac{1}{8} \tau=\zeta$, then the above inequality is equivalent to

$$
\frac{3}{128} b^{+}(\zeta) \geq b^{-}(3-8 \zeta), \quad \zeta \in\left[\frac{1}{4}, \frac{1}{3}\right]
$$

By the definition of $b(t)$, the above inequality holds obviously. It is clear that by $(5.6),\left(H_{4}\right)$ is reasonable.

Step 2 . We show that $\left(H_{5}\right)$ holds. Similar to the arguments in Step 1, letting $c_{1}=1, c_{2}=16$, $\sigma_{2}=\frac{1}{4}, \mu=1$, and $\alpha=1$, then by (5.5) we get

$$
\frac{1}{4} \int_{\frac{1}{4}}^{\frac{1}{3}} G(t, s) a^{+}(s) d s \geq \frac{16}{3} \int_{\frac{1}{3}}^{1} G(t, s) a^{-}(s) d s .
$$

Further, from the above inequality, it follows that

$$
\begin{aligned}
& \frac{1}{4} \int_{\frac{1}{4}}^{\frac{1}{3}} G(t, s) \frac{3}{16} a^{+}(s) d s \geq \int_{\frac{1}{3}}^{1} G(t, s) a^{-}(s) d s \\
& \quad \Leftrightarrow \quad \frac{1}{4} \int_{\frac{1}{4}}^{\frac{1}{3}} G(t, s)\left(\min _{s \in\left[\frac{1}{4}, \frac{1}{3}\right]} G_{1}(s, s)\right) a^{+}(s) d s \geq \int_{\frac{1}{3}}^{1} G(t, s) a^{-}(s) d s \\
& \Rightarrow \frac{1}{4} \int_{\frac{1}{4}}^{\frac{1}{3}} G(t, s) G_{1}(s, s) a^{+}(s) d s \geq \int_{\frac{1}{3}}^{1} G(t, s) a^{-}(s) d s \\
& \Rightarrow \frac{1}{4} \int_{\frac{1}{4}}^{\frac{1}{3}} G(t, s) G_{1}(s, s) a^{+}(s) d s>\frac{1}{3} \int_{\frac{1}{3}}^{1} G(t, s) a^{-}(s) d s .
\end{aligned}
$$

So, $\left(H_{5}\right)$ holds.

Step 3. Similar to the arguments in Step 1, letting $c_{1}=c_{2}=1, \sigma_{3}=\frac{1}{4}, \mu=1$, and $\alpha=\frac{1}{2}$, then $\left(H_{8}\right)$ holds.

Step 4 . It is easy to see by direct calculation that

$$
\int_{0}^{\xi} g^{+}(s) d s=\int_{0}^{\frac{1}{3}} \frac{64}{\sqrt{3}}\left(\frac{1}{3}-s\right) d s=\frac{32}{9 \sqrt{3}}
$$




$$
\begin{aligned}
A^{*} & =\int_{0}^{\xi} \int_{0}^{\xi} G_{1}(s, \tau) a^{+}(s) b^{+}(\tau) d \tau d s=\int_{0}^{\frac{1}{3}} a^{+}(s) \int_{0}^{\frac{1}{3}} G_{1}(s, \tau) b^{+}(\tau) d \tau d s \\
& =\int_{0}^{\frac{1}{3}} a^{+}(s)\left[\int_{0}^{s} \tau(1-s) b^{+}(\tau) d \tau+\int_{s}^{\frac{1}{3}} s(1-\tau) b^{+}(\tau) d \tau\right] d s=\frac{4,325,376}{885,735} .
\end{aligned}
$$

Let $\eta=1, r=2$, and $R=10$. Then we have

$$
\begin{aligned}
& M_{\eta}=\max _{0 \leq x \leq 1}\{\sqrt{x}\}=1, \quad M_{\eta}^{*}=\max \left\{m_{k}^{*}, k=1,2, \ldots, n\right\}=1, \\
& m_{k}=\max _{0 \leq x \leq 1}\{\sqrt{x}\}=1, \quad k=1,2, \ldots, n .
\end{aligned}
$$

Therefore, we get

$$
\begin{aligned}
& \lambda_{1}^{* *}=\frac{\eta}{3 M_{\eta} \int_{0}^{\xi} g^{+}(s) d s} \approx 0.1623, \quad \lambda_{2}^{* *}=\frac{1}{3 A^{*} R} \approx 0.0068, \\
& \lambda_{3}^{* *}=\frac{\eta}{3 n M_{\eta}^{*}} \approx 0.3333 .
\end{aligned}
$$

Hence, all conditions of (II) of Theorem 3.1 hold. Thus by (II) of Theorem 3.1, problem (5.1) admits at least two positive solutions for $0<\lambda_{1} \leq 0.1623,0<\lambda_{2} \leq 0.0068$, and $0<$ $\lambda_{3} \leq 0.3333$.

\section{Acknowledgements}

The authors are grateful to anonymous referees for their constructive comments and suggestions which have greatly improved this paper.

\section{Funding}

This work is sponsored by the National Natural Science Foundation of China (11301178) and the Beijing Natural Science Foundation (1163007).

\section{Availability of data and materials}

Not applicable.

Ethics approval and consent to participate

Not applicable.

\section{Competing interests}

The authors declare that there is no conflict of interest regarding the publication of this manuscript.

\section{Consent for publication}

Not applicable.

Authors' contributions

The authors contributed equally to this article. They have all read and approved the final manuscript.

\section{Publisher's Note}

Springer Nature remains neutral with regard to jurisdictional claims in published maps and institutional affiliations.

Received: 11 January 2018 Accepted: 25 April 2018 Published online: 03 May 2018

\section{References}

1. Onose, H.: Oscillatory properties of the first order nonlinear advance and delayed differential inequalities. Nonlinear Anal. 8, 171-180 (1984)

2. Erbe, L.H., Freedman, H.I., Liu, X.Z., Wu, J.H.: Comparison principles for impulsive parabolic equations with applications to models of single species growth. J. Aust. Math. Soc. Ser. B 32, 382-400 (1991)

3. Pasquero, S.: Ideality criterion for unilateral constraints in time-dependent impulsive mechanics. J. Math. Phys. $\mathbf{4 6}$, 112904 (2005)

4. Guo, Y.: Globally robust stability analysis for stochastic Cohen-Grossberg neural networks with impulse control and time-varying delays. Ukr. Math. J. 69, 1049-1060 (2017) 
5. Nieto, J.J., O'Regan, D.: Variational approach to impulsive differential equations. Nonlinear Anal., Real World Appl. 10, 680-690 (2009)

6. Liu, X., Willms, A.: Impulsive controllability of linear dynamical systems with applications to maneuvers of spacecraft. Math. Probl. Eng. 2, 277-299 (1996)

7. Pasquero, S.: Ideality criterion for unilateral constraints in time-dependent impulsive mechanics. J. Math. Phys. 46, $112904(2005)$

8. Karaca, I.Y., Fen, F.T.: Positive solutions of $n$ th-order $m$-point impulsive boundary value problems. Georgian Math. J. 22, 373-384 (2015)

9. Feng, M., Du, B., Ge, W.: Impulsive boundary value problems with integral boundary conditions and one-dimensional p-Laplacian. Nonlinear Anal. 70, 3119-3126 (2009)

10. Liu, Y., O'Regan, D.: Multiplicity results using bifurcation techniques for a class of boundary value problems of impulsive differential equations. Commun. Nonlinear Sci. Numer. Simul. 16, 1769-1775 (2011)

11. Zhang, X., Feng, M.: Nontrivial convex solutions on a parameter of impulsive differential equation with Monge-Ampère operator. Bound. Value Probl. 2017, 172 (2017)

12. Zhang, H., Liu, L., Wu, Y.: Positive solutions for nth-order nonlinear impulsive singular integro-differential equations on infinite intervals in Banach spaces. Nonlinear Anal. 70, 772-787 (2009)

13. Hao, X., Liu, L., Wu, Y.: Positive solutions for second order impulsive differential equations with integral boundary conditions. Commun. Nonlinear Sci. Numer. Simul. 16, 101-111 (2011)

14. Jiang, J., Liu, L., Wu, Y.: Positive solutions for second order impulsive differential equations with Stieltjes integral boundary conditions. Adv. Differ. Equ. 2012, 124 (2012)

15. Zhang, X., Feng, M., Ge, W.: Existence of solutions of boundary value problems with integral boundary conditions for second-order impulsive integro-differential equations in Banach spaces. J. Comput. Appl. Math. 233, 1915-1926 (2010)

16. Wang, M., Feng, M.: Infinitely many singularities and denumerably many positive solutions for a second-order impulsive Neumann boundary value problem. Bound. Value Probl. 2017, 50 (2017)

17. Chen, X., Du, Z:: Existence of positive periodic solutions for a neutral delay predator-prey model with Hassell-Varley type functional response and impulse. Qual. Theory Dyn. Syst. https://doi.org/10.1007/s12346-017-0223-6

18. Liu, J., Zhao, Z.: Variational approach to second-order damped Hamiltonian systems with impulsive effects. J. Nonlinear Sci. Appl. 9, 3459-3472 (2016)

19. Zhou, J., Li, Y.: Existence and multiplicity of solutions for some Dirichlet problems with impulsive effects. Nonlinear Anal. 71, 2856-2865 (2009)

20. Hao, X. Liu, L., Wu, Y.: Iterative solution for nonlinear impulsive advection-reaction-diffusion equations. J. Nonlinear Sci. Appl. 9, 4070-4077 (2016)

21. Hao, X., Liu, L.: Mild solution of semilinear impulsive integro-differential evolution equation in Banach spaces. Math Methods Appl. Sci. 40, 4832-4841 (2017)

22. Bai, Z., Dong, X., Yin, C.: Existence results for impulsive nonlinear fractional differential equation with mixed boundary conditions. Bound. Value Probl. 2016(1), 63 (2016)

23. Zhang, X., Yang, X., Ge, W.: Positive solutions of $n$ th-order impulsive boundary value problems with integral boundary conditions in Banach spaces. Nonlinear Anal. 71, 5930-5945 (2009)

24. Bai, L., Nieto, J.J., Wang, X.: Variational approach to non-instantaneous impulsive nonlinear differential equations. J. Nonlinear Sci. Appl. 10, 2440-2448 (2017)

25. Tian, Y., Bai, Z:: Existence results for the three-point impulsive boundary value problem involving fractional differential equations. Comput. Math. Appl. 59, 2601-2609 (2010)

26. Zhang, X., Feng, M.: Transformation techniques and fixed point theories to establish the positive solutions of second order impulsive differential equations. J. Comput. Appl. Math. 271, 117-129 (2014)

27. Zuo, M., Hao, X., Liu, L., Cui, Y.: Existence results for impulsive fractional integro-differential equation of mixed type with constant coefficient and antiperiodic boundary conditions. Bound. Value Probl. 2017, 161 (2017)

28. Liu, J., Zhao, Z:: Multiple solutions for impulsive problems with non-autonomous perturbations. Appl. Math. Lett. 64 143-149 (2017)

29. Ma, R., Han, X.: Existence and multiplicity of positive solutions of a nonlinear eigenvalue problem with indefinite weight function. Appl. Math. Comput. 215, 1077-1083 (2009)

30. López-Gómez, J., Tellini, A.: Generating an arbitrarily large number of isolas in a superlinear indefinite problem. Nonlinear Anal. 108, 223-248 (2014)

31. Boscaggin, A., Zanolin, F.: Second-order ordinary differential equations with indefinite weight: the Neumann boundary value problem. Ann. Mat. Pura Appl. 194, 451-478 (2015)

32. Boscaggin, A., Zanolin, F.: Positive periodic solutions of second order nonlinear equations with indefinite weight: multiplicity results and complex dynamics. J. Differ. Equ. 252, 2922-2950 (2012)

33. Sovrano, E., Zanolin, F.: Indefinite weight nonlinear problems with Neumann boundary conditions. J. Math. Anal. Appl. 452, 126-147 (2017)

34. Bravo, J.L., Torres, P.J.: Periodic solutions of a singular equation with indefinite weight. Adv. Nonlinear Stud. 10 927-938 (2010)

35. Wang, F., An, Y:: On positive solutions for a second order differential system with indefinite weight. Appl. Math. Comput. 259, 753-761 (2015)

36. Yao, Q.: Existence and multiplicity of positive radial solutions for a semilinear elliptic equation with change of sign. Appl. Anal. 80, 65-77 (2001)

37. López-Gómez, J., Omari, P., Rivetti, S.: Positive solutions of one-dimensional indefinite capillarity-type problems: a variational approach. J. Differ. Equ. 262, 2335-2392 (2017)

38. López-Gómez, J., Omari, P., Rivetti, S.: Bifurcation of positive solutions for a one-dimensional indefinite quasilinear Neumann problem. Nonlinear Anal. 155, 1-51 (2017)

39. Tokmak, F., Karaca, I.Y.: Existence of symmetric positive solutions for a multipoint boundary value problem with sign-changing nonlinearity on time scales. Bound. Value Probl. 2013, 52 (2013)

40. Karaca, I.Y., Tokmak, F.: Existence of positive solutions for third-order boundary value problems with integral boundary conditions on time scales. J. Inequal. Appl. 2013, 498 (2013) 
41. Wu, Y., Zhao, Z.: Positive solutions for third-order boundary value problems with change of signs. Appl. Math. Comput. 218, 2744-2749 (2011)

42. Jiang, J., Liu, L., Wu, Y: Second-order nonlinear singular Sturm-Liouville problems with integral boundary problems. Appl. Math. Comput. 215, 1573-1582 (2009)

43. Hao, X., Liu, L., Wu, Y.: On positive solutions of $\mathrm{m}$-point nonhomogeneous singular boundary value problem. Nonlinear Anal. 73, 2532-2540 (2010)

44. Liu, W., Liu, L., Wu, Y.: Positive solutions of a singular boundary value problem for systems of second-order differential equations. Appl. Math. Comput. 208, 511-519 (2009)

45. Wang, M., Feng, M.: New Green's function and two infinite families of positive solutions for a second order impulsive singular parametric equation. Adv. Differ. Equ. 2017 (2017) 14 pages

46. Jiang, J., Liu, L., Wu, Y.: Symmetric positive solutions to singular system with multi-point coupled boundary conditions. Appl. Math. Comput. 220, 536-548 (2013)

47. Feng, M:: Multiple positive solutions of four-order impulsive differential equations with integral boundary conditions and one-dimensional p-Laplacian. Bound. Value Probl. 2011, 654871 (2011)

48. Feng, M., Ji, D., Ge, W.: Positive solutions for a class of boundary value problem with integral boundary conditions in Banach spaces. J. Comput. Appl. Math. 222, 351-363 (2008)

49. Guo, D., Lakshmikantham, V:: Nonlinear Problems in Abstract Cones. Academic Press, New York (1988)

50. Zhang, X., Feng, M., Ge, W.: Existence results for nonlinear boundary-value problems with integral boundary conditions in Banach spaces. Nonlinear Anal. 69, 3310-3321 (2008)

51. Zhang, X., Feng, M.: Multi-parameter, impulsive effects and positive periodic solutions of first-order functional differential equations. Bound. Value Probl. 2015, 137 (2015)

\section{Submit your manuscript to a SpringerOpen ${ }^{\circ}$ journal and benefit from:}

- Convenient online submission

- Rigorous peer review

- Open access: articles freely available online

- High visibility within the field

- Retaining the copyright to your article

Submit your next manuscript at $\mathbf{s p r i n g e r o p e n . c o m ~}$ 